\title{
A thermalized nonlinear constitutive model based on contact mechanics
}

\author{
P. Boroumand \\ Vienna University of Technology, Vienna, Austria
}

\begin{abstract}
With the broad industrial applications of curvy-layered structures due to their desirable mechanical properties, contact analysis is no further confined to the single layer half-space media, as originally developed. In the light of this view and along the path towards the mechanical analysis of a thermalized composite structural cable, this paper focuses on the derivation of a normal constitutive "force-deformation" model based on the principles of contact mechanics for layered media in pre-specified temperature scenarios, where both the thermal difference and the heat fluxes are in the game. Applying the Fourier transform method and the Inverse Fourier Convolution algorithm, the final numerical analyses reveal the fact that not only the defined nonlinear spring gets stiffer with a rise in the temperature, but also the deviation from the corresponding layered contact phenomenon in the ambient environment is notably a function of the width of contact.
\end{abstract}

Keywords: thermalization, contact analysis, nonlinear constitutive model.

\section{Introduction}

The principles of contact mechanics originate form the approximation of the curvy mating surfaces by two parabolic functions [1]. Should $R$ be the general arbitrary contacting body radius and $\left(z_{l}, y_{l}\right)$, the horizontal and vertical components of the position vector, Figure 1 shows the customized contact scenario for the current problem of a steel wire $(S)$ in touch with a layered base of Polyethylene $(P E)$ and cement paste (cem). In this figure, $h_{P E}$ and $R_{e q}$ respectively refer to the thickness of the PE layer and the equivalent contact radius, as further clarified.

For $\left(z_{l} / R \ll 1\right)$ and $\cos \left(z_{l} / R\right) \simeq 1-\frac{\left(z_{l} / R\right)^{2}}{2}$, "Hertz" used the geometrical condition, defined by eqn (1) to relate the relative deformation of any two curved 
contact scenario for the current problem of a steel wire $(S)$ in touch with a layered base of Polyethylene $(P E)$ and cement paste $(\mathrm{cem})$. In this Figure,

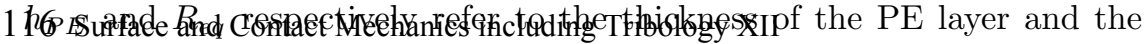
equivalent contact radius, as further clarified.
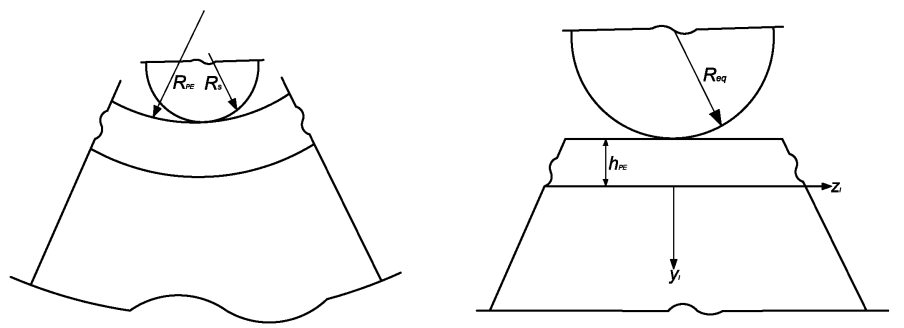

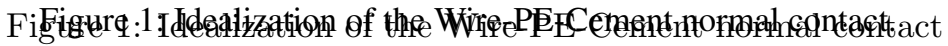

For $\left(z_{l} / R<<1\right)$ and $\cos \left(z_{l} / R\right) \simeq 1-\frac{\left(z_{l} / R\right)^{2}}{2}$, "Hertz" used the geo-

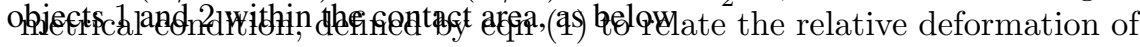
any two curved objects 1 and 2 within the contact area, as below

$$
\begin{gathered}
\bar{v}_{1}+\bar{v}_{2}+y_{l_{1}}+y_{l_{2}}=\delta_{1}+\delta_{2}, \bar{v}+y=\delta \\
\bar{v}_{1}+\bar{v}_{2}+y_{l_{1}}+y_{l_{2}}=\delta_{1}+\delta_{2}, \bar{v}+y=\delta
\end{gathered}
$$

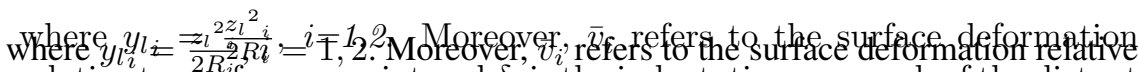

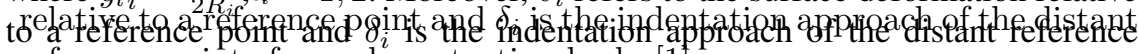

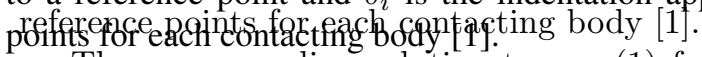

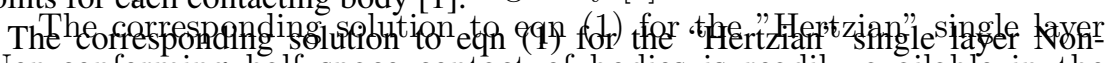

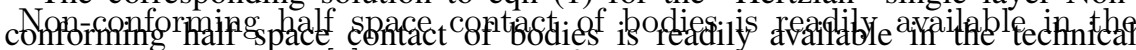

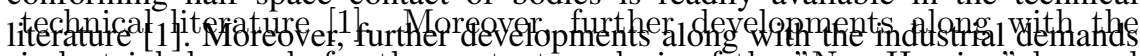

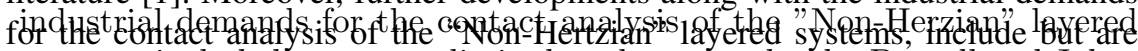

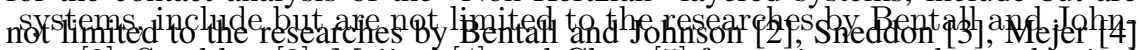

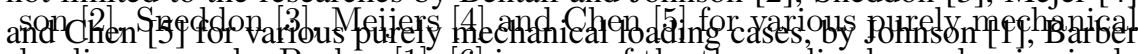

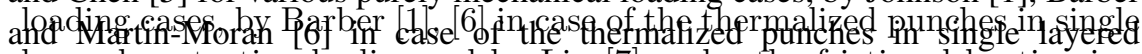

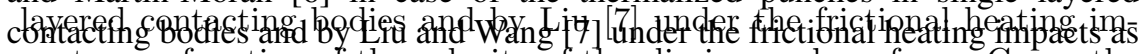

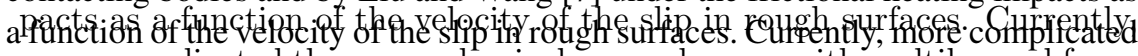

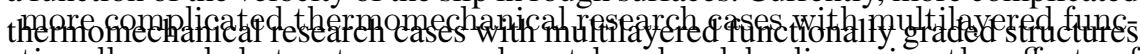

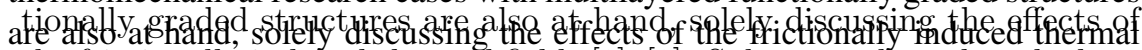

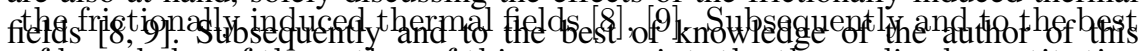

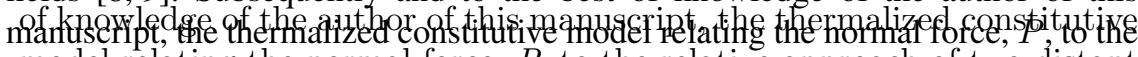

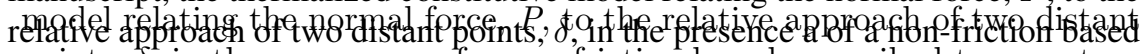

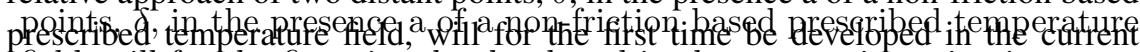
investigattlon.

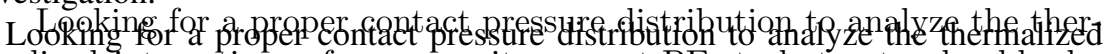

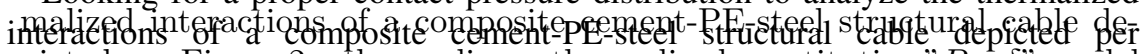

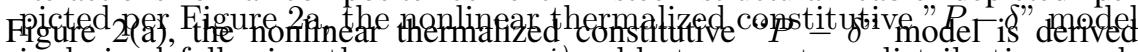

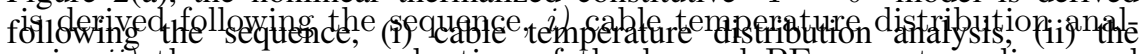

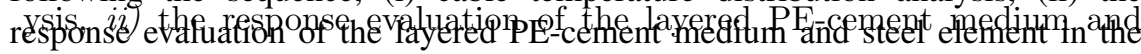
frequency and back into the space domain and eventually, (iii) the numerical solution to the derived system of equations for the distributed contact pressure evaluation for three different temperature regimes detected in the cable. Results and conclusions are briefly discussed in the final sections, as well. 


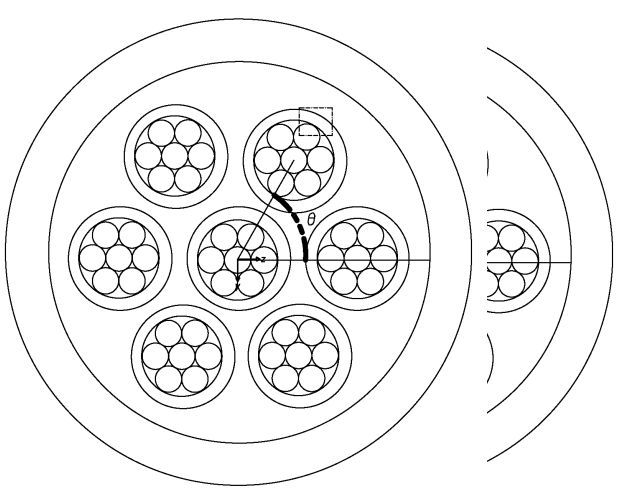

(a)

(a)

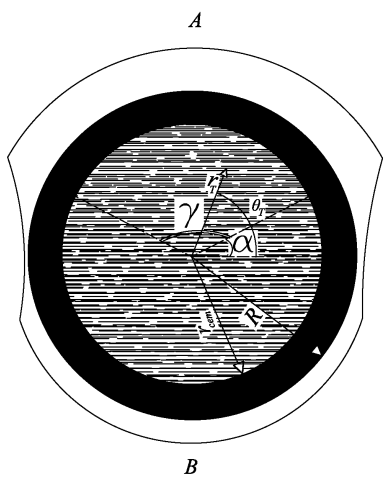

(b)

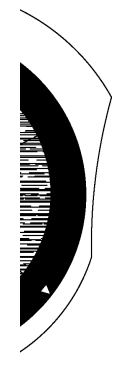

(b)

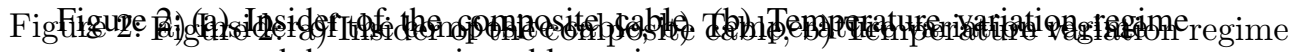

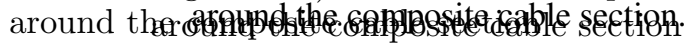

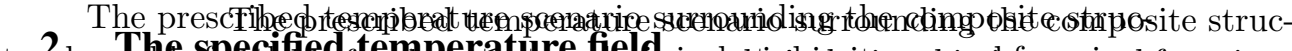

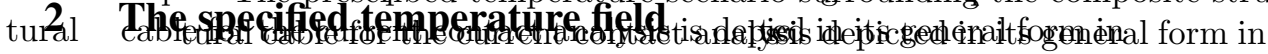

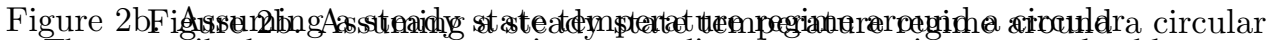

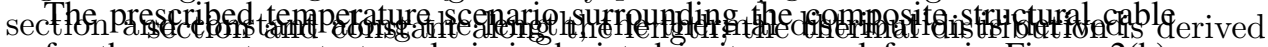

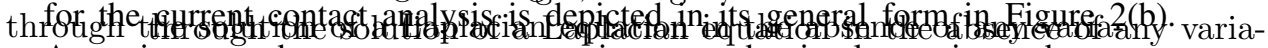

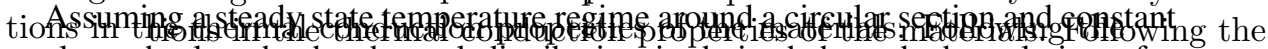

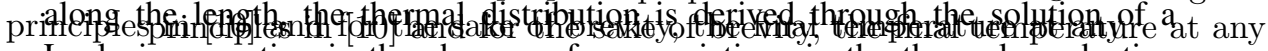

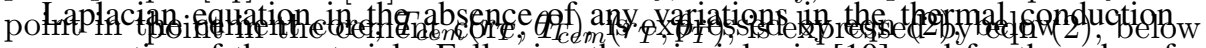

properties of the materials. Following the principles in [10] and for the sake of

brevity, the final temperature at any point in the cement core, $T_{\text {cem }}\left(r_{T}, \theta_{T}\right)$, is

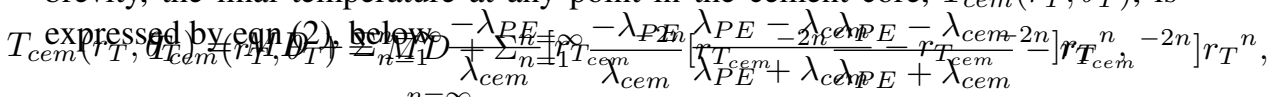

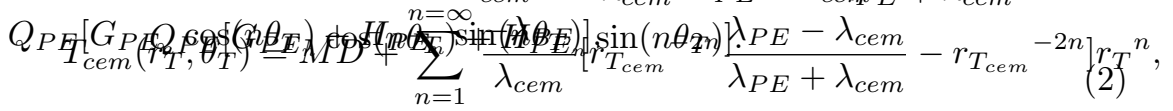

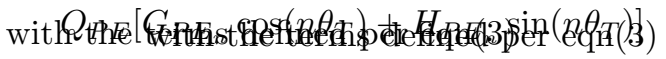

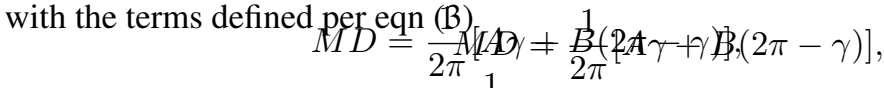

$$
\begin{aligned}
& M D=\frac{1}{2 \pi}[A \gamma+B(2 \pi-\gamma)], \\
& Q G_{n}=\frac{1}{n \pi}\left[r_{T_{c e m}}{ }^{-2 n} \frac{\lambda_{P E}-\lambda_{c e m}}{\lambda_{P E}+\lambda_{c e m}} R^{n}+R^{-n}\right]^{-1} \\
& \{(B-A) \sin (n \alpha)+(A-B) \sin [n(\gamma+\alpha)]\}, \\
& Q H_{n}=-\frac{1}{n \pi}\left[r_{T_{c e m}}{ }^{-2 n} \frac{\lambda_{P E}-\lambda_{c e m}}{\lambda_{P E}+\lambda_{c e m}} R^{n}+R^{-n}\right]^{-1} \\
& \{(B-A) \cos (n \alpha)+(A-B) \cos [n(\gamma+\alpha)]\}
\end{aligned}
$$


where conductivity of the materials is represented by $\lambda$. In addition, $r_{T}$ is the radius of any arbitrary point within the circular core and $\theta_{T}$, the corresponding polar angle as shown in Figure 2(b). Moreover, $r_{T_{c e m}}$ and $R$ are the total radius of the cement core and the composite section, respectively and $n$ is a natural number. The upper and lower parts temperatures are presented by $A$ and $B$ around the cable with the distinguished angles $\alpha$ and $\gamma$ in the same Figure 2(b).

Although the equations above determine the temperature field within the cement core, it is assumed that the internal PE layer and the surrounded steel wires temperature in Figure 2(a) will remain constant and equal to an average value of the immediately confining cement paste.

\section{Steel-PE-cement interactions}

The mathematical formulations towards appropriate thermalized " $P-\delta$ " model derivation for the steel-PE-cement layered structure per Figure 1, will be developed and discussed, as follows.

\subsection{Mathematical formulation of a semi-infinite layered static response}

Generally, determination of the stress, strains and the corresponding displacements within a continuum medium requires the satisfaction of the equilibrium and compatibility equations, as well as the applied boundary conditions or equivalently, the satisfaction of the single Airy stress function which takes the following form in the presence of thermal fluctuations [11].

$$
\nabla^{4} \phi+\nabla^{2}\left(E \alpha_{T} \Delta T\right)=0
$$

In eqn (4), $\phi$ is the Airy stress function and $E$ and $\alpha_{T}$ respectively represent the modulus of elasticity and the coefficient of linear thermal expansion. In addition, $\Delta T$ refers to the thermal deviation from the reference temperature, $T_{r e f}=23^{\circ} \mathrm{C}$.

Accordingly and due to the layered contact scenario shown in Figure 1, the solution to eqn (4) should meet the equality of the normal and shear tractions at both the interface of the PE-cement layers and the contacting face with the steel elements, as well as the match of the horizontal and vertical components of the displacement at the joint PE-cement surface. An ultimate boundary condition has to guarantee the boundness of the cement infinite layer vertical deformation as $y_{l} \longrightarrow \infty$. Eventually, the solution to the biharmonic eqn (4) for the semi-infinite PE-cement layered media confined at the contacting surface, is best described by the application of Fourier transform and seeking the response in the frequency domain $\omega[3]$.

\subsubsection{Solution in the frequency domain}

Based on the explanations in the preceding subsection, the explicit application of Fourier transform to the biharmonic Airy function takes the form of eqn (5) with the "^" symbol indicating the transformed version of the function in the frequency 
domain. The solution to this 4 th order nonhomogeneous equation given below for each layer, consists of a general and a particular part $\left(\hat{\phi}_{P}\right)$, guaranteeing the finiteness of the response $[3,12]$

$$
\hat{\phi}\left(y_{l}, \omega\right)=e^{|\omega| y_{l}}\left(C_{1}+C_{2}\right)+e^{-|\omega| y_{l}}\left(C_{3}+C_{4}\right)+\hat{\phi}_{P}\left(y_{l}, \omega\right)
$$

with $C_{i}, i=1,4$ being the constants of integration, which will further be derived by the satisfaction of the previously defined boundary conditions.

In transforming the boundary conditions into the frequency domain, it is well known that [11]

$$
\sigma_{z_{l}}=\frac{\partial^{2} \phi\left(y_{l}, z_{l}\right)}{\partial y_{l}^{2}}, \quad \sigma_{y_{l}}=\frac{\partial^{2} \phi\left(y_{l}, z_{l}\right)}{\partial z_{l}^{2}}, \quad \tau_{y_{l} z_{l}}=-\frac{\partial^{2} \phi\left(y_{l}, z_{l}\right)}{\partial z_{l} \partial y_{l}}
$$

with $\sigma_{z_{l}}, \sigma_{y_{l}}$ and $\tau_{y_{l} z_{l}}$, being the normal and shear components of stresses in the indexed directions, accordingly.

Replacing the corresponding elasticity terms for the left-hand side of eqn (6), applying the Fourier transform to this set of equations with $F\left[f^{(n)}(x)\right]=$ $(i \omega)^{n} \hat{f}(\omega)$ and simultaneous solution to the derived expressions after some manipulations will render the following term for the vertical component of the displacement in the frequency domain with $\nu$, being the Poisson's ratio [11]

$$
\hat{v}=\frac{\left(1-\nu^{2}\right)}{E \omega^{2}} \frac{\partial^{3} \hat{\phi}\left(y_{l}, z_{l}\right)}{\partial y_{l}{ }^{3}}+\frac{(1+\nu)(\nu-2)}{E} \frac{\partial \hat{\phi}\left(y_{l}, z_{l}\right)}{\partial y_{l}}+\frac{(1+\nu)}{\omega^{2}} \frac{\partial\left(\alpha_{T} \Delta T\right)}{\partial y_{l}}
$$

all the other terms hold their definitions as expressed earlier.

Eventually, as eqn (7) is still undetermined in terms of $\phi\left(y_{l}, z_{l}\right)$ and the corresponding coefficients, application of the earlier defined displacement boundary conditions will determine $C_{i j}, i=1,4, j=P E$, cem for each layer. However, the details could not explicitly be covered in this manuscript due to the page limitations [3].

Furthermore, with the numerically negligible contributions $\left(\mathcal{O}^{-10}\right)$ of the particular solution, $\hat{\phi}_{p}$, raised by the term $\nabla^{2}\left(E \alpha_{T} \Delta T\right)$ in eqn (4), only the thermal effects on the coefficients in the general part of the response is taken into account. Subsequently, a MATLAB code is set up to solve the derived system of linear equations for the unknown coefficients $C_{i}, i=1,4$ in the PEcement arrangement, and further return the displacement of these two layers in the frequency domain, using eqn (7).

\subsubsection{Inverse Fourier transform}

Once eqn (7), restated per eqn (8) is explicitly derived in terms of the $C_{i j}, i=$ $1,4, j=P E$, cem in the frequency domain, the second phase of calculations has to concentrate on the derivation of the normal displacement of the contacting bodies back in the space domain. To maintain the calculations efficiency in eqn (9), the inverse Fourier convolution scheme is further applied, as symbolically 
represented by the expressions, below

$$
\begin{gathered}
\hat{v}(\omega)=H_{p}(\omega) \hat{p}(\omega)+H_{q}(\omega) \hat{q}(\omega)+H_{\Delta T}(\omega) \hat{\Delta T}+H_{\Delta^{\prime} T}(\omega) \frac{\partial\left(\hat{\alpha}_{T} \Delta T\right)}{\partial y_{l}} \\
v\left(z_{l}\right)=F^{-1}[\hat{v}(\omega)]=\frac{1}{2 \pi} \int_{-\infty}^{+\infty} \hat{v} e^{i \omega z_{l}} d \omega \\
=\int_{-\infty}^{+\infty}\left\{F^{-1}\left[H_{p}\right]\left(z_{l}-\zeta\right) p(\zeta)+F^{-1}\left[H_{q}\right]\left(z_{l}-\zeta\right) q(\zeta)\right\} d \zeta \\
+\int_{-\infty}^{+\infty}\left\{F^{-1}\left[H_{\Delta T}\right]\left(z_{l}-\zeta\right) \Delta T(\zeta)+F^{-1}\left[H_{\Delta^{\prime} T}\right]\left(z_{l}-\zeta\right) \frac{\partial\left(\alpha_{T} \Delta T\right)}{\partial y_{l}}\right\} d \zeta
\end{gathered}
$$

with $F^{-1}$ being the inverse Fourier transform operator, $\zeta$, the dummy convolution variable in the space domain and $H_{i}(\omega), i=p, q, \Delta T, \Delta T$, the Green's functions due to the pressure, shear, temperature and heat flux effects in the frequency domain. All the other symbols maintain the same definition as earlier described. In the next step, the transform in eqn (10) for the PE-cement layer will be entailed. Nevertheless, due to the generally reported negligible coupled shear and normal effects in contact studies, shear Green's function will be removed from the analysis, as it proceeds [1].

Overall, to numerically work out eqn (10) for the Green functions in the frequency domain, a trapezoidal rule is applied. However, unlike the even Green functions, $H_{i}(\omega), i=\Delta T, \Delta^{\prime} T$ in the PE and cement layers, $H_{p}(\omega)$ is integrated numerically by trapezoids at suitably small intervals up to a sufficiently large value, $\omega_{0}=1600$, and the rest by the application of cosine integrals, symbolized by Cosint, as detailed per eqn (11) [2,12-14].

$$
\begin{aligned}
F^{-1}\left[H_{p_{P E}}(\omega)\right] & =\frac{1}{\pi} \int_{0}^{+\infty} H_{p_{P E}}(\omega) \cos \left(\omega z_{l}\right) d \omega \\
& =\frac{1}{\pi}\left\{\Sigma_{\omega=0}^{\omega_{0}} H_{p_{P E}}(\omega) \cos \left(\omega z_{l}\right)+\frac{2\left(\nu_{P E}^{2}-1\right)}{E_{P E}} \operatorname{Cosint}\left(\omega_{0} z_{l}\right)\right\}
\end{aligned}
$$

Before proceeding to the contributions by the steel wire element, it is reminded that similar numerical integration schemes towards the derivation of the $F^{-1}\left[H_{i}(\omega)\right], i=p, \Delta T, \Delta^{\prime} T$ functions at the common surface of the PE and cement layer, being the reference point of indentation in the layered phase, must be followed as well. However, the explicit mathematical details are avoided for the sake of brevity, yet, the vertical displacement of the joint surface will appear as $v_{a t t}$ in further notations. 
Eventually, the derivation of the total vertical deformation in the space domain through the inverse Fourier convolution is discussed in the following subsection.

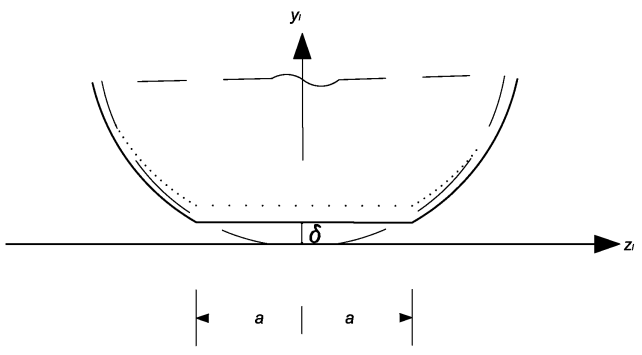

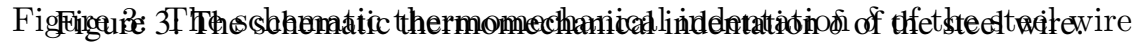

\section{$3_{3.3}$ Confact of a thermalized elastic steel cylinder}

As long as the steel element is only a single layer medium significant The nathematical steps remained towards the derivation of the desired simplifications through the application o, the alreatition of the general solutions in the frequency domain. could be achieved. By the cancelation of al solutions in the rrequency domain could be achieved. By the cancelation of an the contributions by the heat flux term, steel, the only required modification to the mechanically induced deformation could be captured by the effects of a constant $\Delta T$, superposed on the impacts of the contact stress $\underline{p}_{v}$. The theoretical formulations below serye as the mathematical representation of the mechanical contributions by a unit $p$ in the frequency domain. where

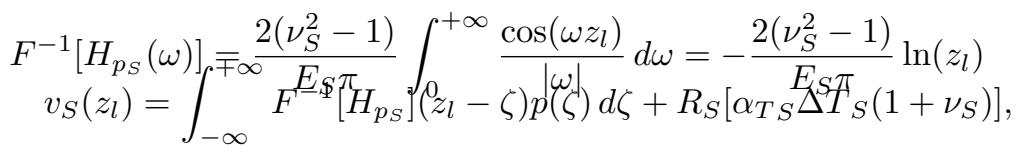

where all the terms are defined earlier elsewhere with the index $S$ for the steel.

To handle the temperature effects pictured in Figure 3 , it must be noticed that a positive temperature change will induce a ${ }^{2} p_{S}$ surface displacement in the negative direction and the superposition of the thermal and mechanical stresses will be with the subscripts att and $S$ labeling the corresponding parameters for of a destructive type unless $\Delta T$. Furthermore, since the actual undeformed shape of the wire and the constant temperature rise within the object is known, the appearance. As stated fefore, in eon 14 the free thermal expansion of the therearance. Astions could separately and readily be added to the mechanical one therma deformations could separately and.readily be added to the mechanical one right in the space domain, following linear thermoelasticity. The final indentation effects and $v_{S C}\left(R_{f}\right.$ ) rspresents the deformation of the wire reference point of the steel wire surface shown by $\&$ per Figure 3 is the one capturing the effect of All the other variables hold their.definitions, as detined earlier in the text. the added thermal part (continuous line) to the mechanically induced one (dotted line Finally, the only remained step is to derive the single unknown of the line)blem per eqn (13) being the thermalized contact pressure distribution.

Similar to the deformation of the PE layer, the surface deformation of the thermalized steel element has to be measured relative to a reference point, being the center of the contacting wire. For the transparency of the future calculations, $F^{-1}\left[H_{p_{S C}}(\omega)\right]$ will symbolize the deformation of the center of the steel wire at a radius distance, $R_{S}$, due to the mechanical stresses by eqn (12).

Eventually, the derivation of the total vertical deformation in the space domain through the inverse Fourier convolution is discussed in the following subsection. 


\subsection{Total inverse Fourier convolution}

The mathematical steps remained towards the derivation of the desired thermalized constitutive model " $P-\delta$ " is the substitution of the general eqn (9), customized separately for the contributing elements steel, PE and the cement paste, into the geometrical contact condition represented by eqn (1). Hence, the substituted eqn (13) is given below

$$
\bar{v}+y=v_{S}\left(z_{l}\right)-v_{S C}\left(R_{S}\right)+v_{P E}\left(z_{l}\right)-v_{a t t}(0)+y=\delta
$$

where

$$
\begin{gathered}
v_{S}\left(z_{l}\right)=\int_{-\infty}^{+\infty} F^{-1}\left[H_{p_{S}}\right]\left(z_{l}-\zeta\right) p(\zeta) d \zeta+R_{S}\left[\alpha_{T S} \Delta T_{S}\left(1+\nu_{S}\right)\right] \\
v_{S C}\left(R_{S}\right)=\int_{-\infty}^{+\infty} F^{-1}\left[H_{p_{S C}}\right]\left(R_{S}-\zeta\right) p(\zeta) d \zeta
\end{gathered}
$$

with the subscripts att and $S$ labeling the corresponding parameters for the PEcement attachment interface and the steel wire, in the order of appearance. As stated before, in eqn (14) the free thermal expansion of the steel element in a plane strain state is directly superposed to the mechanical effects and $v_{S C}\left(R_{S}\right)$ represents the deformation of the wire reference point. All the other variables hold their definitions, as defined earlier in the text.

Finally, the only remained step is to derive the single unknown of the problem per eqn (13), being the thermalized contact pressure distribution, $p$, at the mating surface of steel and the layered medium. To simplify the final numerical integration solution, first the displacement of an arbitrary point, being the ultimate width of contact, $z_{l}=a$, is subtracted from each side of the equation, as in eqn (15)

$$
v_{S}\left(z_{l}\right)+v_{P E}\left(z_{l}\right)-v_{S}(a)-v_{P E}(a)=\frac{\left(a^{2}-z_{l}^{2}\right)}{2 R_{e q}}
$$

with

$$
y=y_{l_{S}}+y_{l_{P E}}=\frac{z_{l}^{2}}{2 R_{S}}+\frac{z_{l}^{2}}{2\left(-R_{P E}\right)}=\frac{z_{l}^{2}}{2 R_{e q}}
$$

with all the terms defined earlier.

As follows, the outcome of the computational procedure will be discussed.

\section{Results and discussion}

At last, the solution to the derived set of $(n-1)$ independent linear equations for $n$ segments provided by eqn (15) is numerically followed for the three thermal regions, identified in the cable section and tabulated by $\Delta T_{c e m}$ and $\frac{\partial\left(\alpha_{T} \Delta T\right)}{\partial y_{l}}$ per Table 1. In this table, the reproduced numerical values represent the thermal characteristics of the point at the origin in the PE-cement attachment, 
Table 1: The cable distinguished thermalized regions.

\begin{tabular}{|l|l|l|}
\hline$\theta^{\circ}$ & $\Delta T^{\circ} \mathrm{C}$ & $\Delta^{\prime} T \frac{{ }^{\circ} \mathrm{C}}{m}$ \\
\hline 60 & 36 & $+4.6841 \times 10^{2}$ \\
\hline 0 & 15 & $-3.1389 \times 10^{2}$ \\
\hline 300 & 6 & $-2.5645 \times 10^{2}$ \\
\hline
\end{tabular}

per Figure 2(a) for different values of the angle $\theta$. Furthermore and based on the provided numerical data, attention has to be paid to the fact that the contact characteristics are not only influenced by the value of the temperature deviation, $\Delta T$, but also vary as a function of the value as well as the sign of its derivative, $\Delta^{\prime} T$. Mathematically, such a phenomenon manifests itself by affecting the right hand side of the contact eqn (13) while solving for the unknown pressure, $p$.

Next, for the sake of comparison, the normalized pressure profile for the layered set up in eqn (15) and the normalized "Hertzian" pressure distribution corresponding to the contact of a steel wire with a semi-infinite PE layer by eqn (17), is depicted in Figure 4 for $\Delta T=15^{\circ} \mathrm{C}$ [1]. All the diagrams have been normalized to the maximum pressure in a Hertzian contact $\left(p_{H 0}\right)$ of the same width, $a$, by eqn (17). As observed, unlike a single layer purely "Hertzian" contact, the normalized pressure profile shows some dependencies on the width of contact. According to the data per Figure 4, the deviation from a "Hertzian" pressure distribution case gets more emphasized with an increase in the width of contact for the current mechanical properties of the mating objects. Similar width dependent behavior of the pressure profile in the purely mechanical contact of layered media has been previously reported by pioneering investigators, as well $[4,14]$. The results presented in the current manuscript were also verified by the comparison of a nonthermalized case data with those reported by Mejer and Gupta to reassure the numerical accuracy of the set up programs which due to the available space limitations, are omitted [4, 14].

$$
p_{H}\left(z_{l}\right)=\frac{2 P}{\pi a^{2}}\left(a^{2}-z_{l}^{2}\right)^{\frac{1}{2}}
$$

Once the pressure profiles for the different contacting widths are derived, a back substitution into eqn (13) will consequently render the required approach of the contacting bodies, $\delta$. The results of the aforementioned numerical procedure for three different temperature regimes in addition to a nonthermalized layered case have been depicted for $R_{S}=h_{P E}=2.25 \mathrm{~mm}$ in Figure 5(a). As noticed, the derived " $P-\delta$ " curves reveal the point that not only the temperature variation causes a shift to the nonthermalized " $P-\delta$ " relation, but also it changes the stiffness of the equivalent spring, in a nonlinear scheme. Furthermore, 


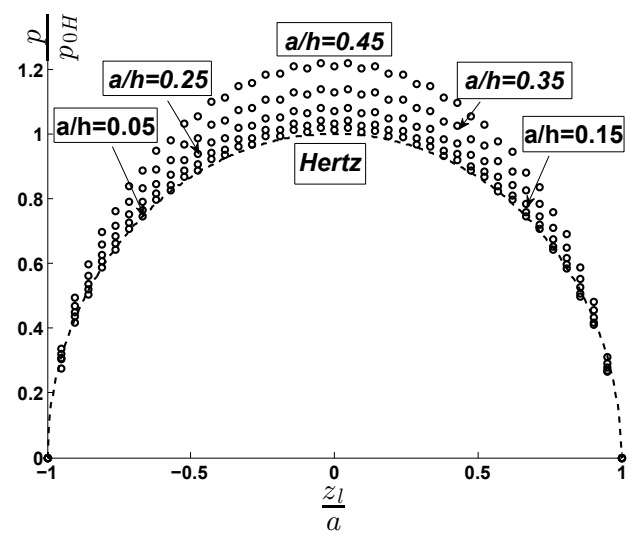

Figure 4: Normalized "Hertzian" and the derived "Non-Hertzian" contact pressure profile for $\Delta T=15^{\circ} \mathrm{C}$.

Figure 4: Normalized "Hertzian" and the derived "Non-Hertzian" contact pressype profile for $\Delta T=15^{0} \mathrm{C}$

${ }_{10}^{12 \times 10^{4}}$ Once the pressure profiles the different contacting widths are derived, a back substitution rint eqn (139) will consequently render the re-

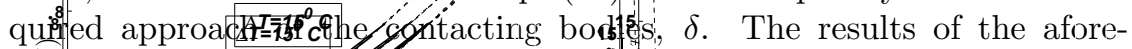

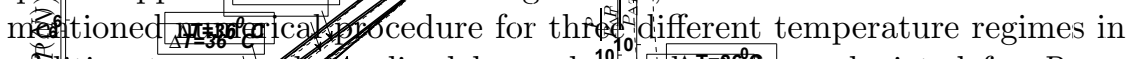

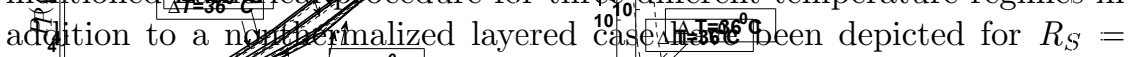

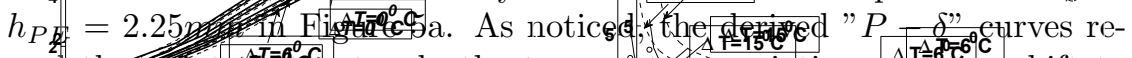
vea torint the temperation

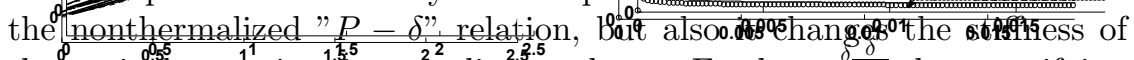

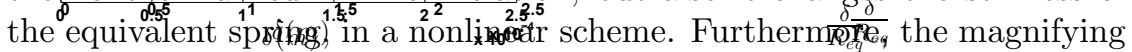

(a)

(b)

Figure 5: (a) Nonlinear thermalized " $P-\delta$ " relationship and the fitted parabolic functions. $($ a $)$ (b) Normalized force ratio vs. the (b) $(0)$ )ormalized contact

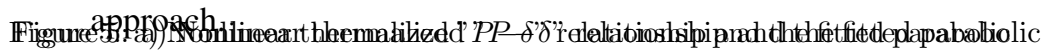

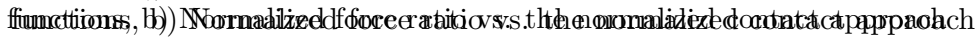

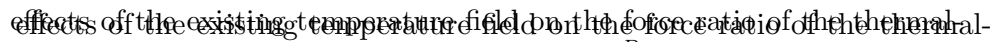

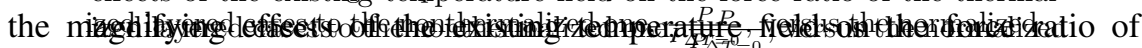

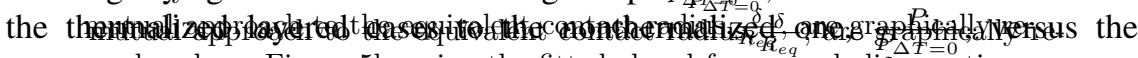

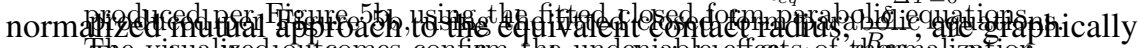

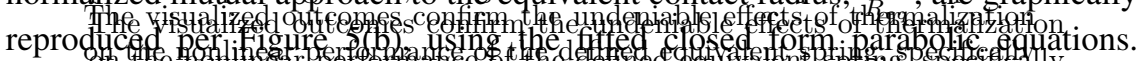
The what the nonlinear performance of the defined equivalent spring, specifically with a decrease in the contact width and/or the contact approach.

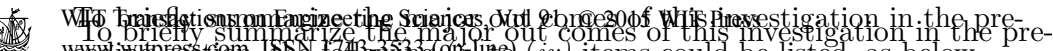

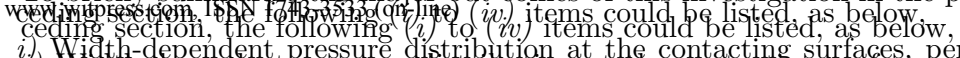
i.) Width-dependent pressure distribution at the contacting surfaces, per Figure 4 Pressure distribution deviates more significantly from a "fterzian 


\section{Conclusion}

To briefly summarize the major out comes of this investigation in the preceding section, the following (i) to (iv) items could be listed, as below.

(i) Width-dependent pressure distribution at the contacting surfaces, per Figure 4. Pressure distribution deviates more significantly from a "Hertzian" one, as the width of contact increases.

(ii) A shift in the constitutive model in the layered thermalized cases in comparison to the layered nonthermalized case (Figure 5(a)).

(iii) The nonlinear variation of the constitutive model itself for the different numerically calculated thermal cases, per the same Figure 5(a).

(iv) More significant deviations from a nonthermalized layered " $P-\delta$ " relation for lower values of approach of the two mating bodies, $\delta$, the subject of Figure 5(b).

\section{References}

[1] Johnson, K.L., Contact Mechanics. Cambridge Univ. Pr.: Cambridge, 1989.

[2] Bentall, R.H. \& Johnson, K.L., An elastic strip in plane rolling contact. International Journal of Mechanical Science, 10, pp. 637-668, 1968.

[3] Sneddon, I.N., (ed.) Fourier Transforms. McGraw-Hill: New York, 1951.

[4] Mejer, P., The contact problem of a rigid cylinder on an elastic layer. Applied Science Research, 18, p. 353, 1968.

[5] Chen, W.T., Computation of stresses and displacements in a layered elastic medium. International Journal of Engineering Science, 9, p. 775, 1971.

[6] Barber, J.R. \& Martin-Moran, C.J., Green's functions for transient thermoelastic contact problems for the half-plane. Wear, 79, pp. 11-19, 1982.

[7] Liu, G. \& Wang, Q., Thermoelastic asperity contacts, frictional shear, and parameter correlations. Tribology, 122, pp. 300-307, 200.

[8] Liu, J., Ke, L.L. \& Wang, Y.S., Two-dimensional thermoelastic contact problem of functionally graded materials involving frictional heating. International Journal of Solids and Structures, 48, pp. 2536-2548, 2011.

[9] Afferrante, L. \& Ciavarella, M., Separated steady state solutions for two thermoelastic half-planes in contact with out-of-plane sliding. the Mechanics and Physics of solids, 53, pp. 1449-1475, 2005.

[10] Noda, N., Hetnarski, R.B. \& Tanigawa, Y., Thermal Stresses. LASTRAN: Rochester, 2000.

[11] Ugural, A.C. \& Fenster, S.K., Advanced Strength and Applied Elasticity. Elsevier: New York, 1981.

[12] Greenberg, M.D., Advanced Engineering Mathematics. Prentice-Hall: New Jersey, 1988.

[13] MATLAB, 8.0.0.783 (R2012b). The MathWorks Inc.: Natick, Massachusetts, 2014.

[14] Gupta, P.K. \& Walowit, J.A., Contact stresses between an elastic cylinder and a layered elastic solid. Lubrication Technology, pp. 250-257, 1974. 